\title{
Healthcare in overview across the globe
}

\section{THE GERMAN HEALTHCARE SYSTEM}

\section{Döring A, Paul F}

NeuroCure Clinical Research Center, Charité-Universitätsmedizin Berlin, Germany

Germany's healthcare system is a contribution-based social insurance model, whose main features can be traced back to the beginning of the Middle Ages and even earlier. It is an important economic area and, with its large workforce of approximately 4.3 million employees (status in 2007), also plays a key role in labor market policy.
In its current form, the German healthcare system is extremely complex and which even experts find difficult to grasp. The foundation of Germany's healthcare system is derived from Germany's Basic Law (Grundgesetz), which obliges the state to provide social services to its citizens (Article 20, 28, of the Basic Law). Specifically, the state must ensure sufficient, needs-based ambulatory and inpatient medical treatment, in qualitative and quantitative terms, as well as guarantee the provision of medicine. The federal government may assume this duty itself or delegate it to state governments and institutions in the form of service guarantee contracts $(\S 72$, German Social Insurance Code, Book V).

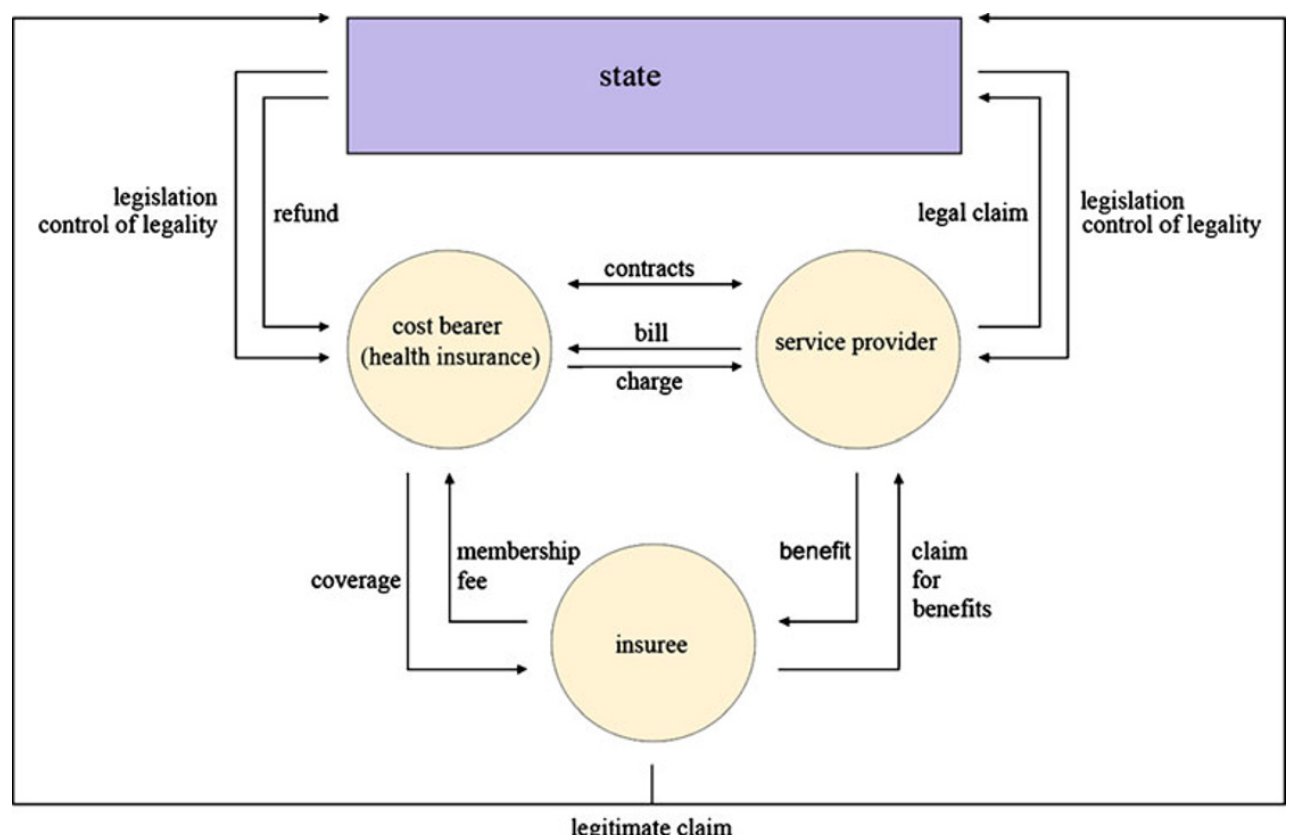

Fig. 1. The structure of the German healthcare system [1]

Basic principles of social rights are used as the framework for ensuring social security in cases of illness, and must be followed by both the health insurance companies and health service providers. The principle of the welfare state is based on the Federal Republic of Germany's Basic Law (Grundgesetz), which specifies that the state must guarantee all citizens social 
justice and the equal participation in society, including appropriate treatment in case of illness. Individuals with below average earning power or economic resources must receive the same quality and quantity of medical care as others. Thus, overall, the healthcare system is organized around the principle of solidarity (Solidarprinzip), which provides that every member of a supportive society is entitled to assistance from the other members of the society in the case of illness. A main feature of the statutory health insurance scheme is social reconciliation (Solidarausgleich), which is expressed in two main ways: firstly, between the healthy and ill, in that all members-including the healthy-meet the costs of the necessary treatment and benefits if a member falls ill regardless of the individual's economic resources, including the additional costs of securing the individual's livelihood, such as continued wages and sickness benefits; and secondly, between high and low incomes, in that up to a defined income threshold, all members of the statutory health insurance pay an incomedependant percentage of their earnings. This solidarity model stands in contrast to the subsidization principle, which provides that an individual's expenses are only then assumed by the supportive society if the individual is demonstrably overextended. Until such a point, the individual is obliged to contribute to the treatment costs. In the subsidization model, the healthcare expenses are met by a sequence of supportive societies from smallest to largest: the individual, his or her martial or life partner, the family, the statutory health insurance fund, and finally the services the individual is entitled to as a citizen.

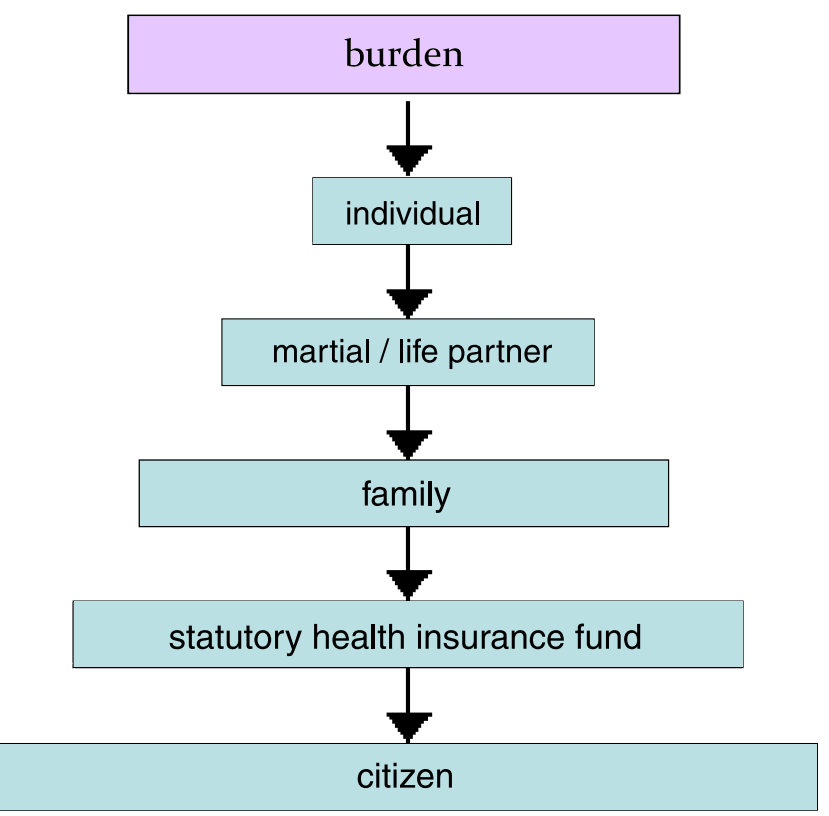

Fig. 2. The subsidization model [1]
The aim is to give an overview about the German healthcare system and gain an understanding of its complexity and principles of organisation. Please find more information about the German healthcare system on the EPMA World Congress 2011 in Bonn, Germany.

\section{References:}

1. Simon, M. Das Gesundheitssystem in Deutschland. Eine Einführung in Struktur und Funktionsweise. 3rd edition, Hans Huber; 2010.

\section{HEALTHCARE IN THE U.K.}

\section{Grosios K}

University of Leeds, UK

The UK healthcare system, National Health Service (NHS), came into existence in the aftermath of the Second World War under the principles of universality, free at the point of delivery, equity, and paid for by central funding. It has evolved to become one of the largest healthcare systems in the world. The Health Act 2009 established the "NHS Constitution" which formally brings together the purpose and principles of the NHS in England, its values, as they have been developed by patients, public and staff and the rights, pledges and responsibilities of patients, public and staff. Scotland, Northern Ireland and Wales have also agreed to a high level statement declaring the principles of the NHS across the UK, even though services may be provided differently in the four countries, reflecting their different health needs and situations. The health research strategy of the NHS is being implemented through National Institute of Health Research (NIHR) and in 1999 the National Institute for Health and Clinical Excellence (NICE) was established as the body responsible for developing national guidelines and standards related to, health promotion and prevention, assessment of new and existing technology (including medicines and procedures) and treatment and care clinical guidance, available across the NHS.

In 2010 the publication of the White Paper "Equity and excellence: Liberating the NHS" heralded one of the biggest shake ups of the health system since its inception. Through the paper the coalition government announced its strategy on how it will "create a more responsive, patient-centred NHS which achieves outcomes that are among the best in the world". Since then plans for reshaping the system have faced strong criticism and changes remain undefined and unimplemented. Nevertheless, the development of preventive, people-centred (shared decision-making/ no decision about me without me) and more productive healthcare services is being seen as key 
for the NHS to respond to the challenges that all modern healthcare systems are facing in the $21^{\text {st }}$ century.

Predictive, preventive and/or personalised medicine (PPPM) services within the NHS have traditionally been offered and are part of disease diagnosis and treatment. Preventive medicine is its own established entity and relevant services, such as cancer screening, child health promotion and immunisation programmes, are directed by Public Health and offered either via GP, community services or hospitals. References to predictive and personalised medicine are increasingly being introduced in NHS related information and personalised therapies in the form of diagnostics and drug therapies are commonly prescribed and used and are increasingly available via commercial/private non-NHS routes. Implementation of PPPM interventions may provide solutions to many of the challenges that healthcare in the UK is facing. This is in turn creating a new way of thinking and set of standards and generates new dilemmas and issues that need to be resolved considering multiple aspects such as disease and health evolution and scientific understanding, professionals and public expectations and education, technological advancement and wider health policies, regulations and financial environment.

\section{HEALTHCARE OVERVIEW: EUROPEAN AREA \& GLOBAL SCALE \\ Iso $H$ \\ National Representative of EPMA in Japan}

Healthcare for cardiovascular prevention and treatment in Japan is overviewed because the decline in cardiovascular disease in particular stroke since the 1960s has contributed to achieve one of the longest life expectancies in the world. The national prevention program for cardiovascular disease was launched in 1982 under the Health Service for the Elderly Act based on the establishment of national health insurance coverage since 1961. Under this Act, insurers are municipal governments and are financed with help from prefectural and national governments to conduct health screening and health education for residents aged 40 and over, as well as care for the aged. The Industrial Safety and Health Act since 1972 has assured health screening and education for company employees. The universal insurance coverage and health screening and education system enhanced the hypertension control and lifestyle modification for highrisk individuals in company and community settings.

Meanwhile, the national government performed a series of revisions to the Medical Care Act in 1985, 1992, 1997, and 2000 to control the number of beds, to differentiate hospital roles as high-technology hospitals, long-term care hospitals, and regional tertiary hospitals, to emphasize the importance of informed consent and staff training, and to enhance amenities. That act has improved the operation of local ambulance system that allowed patients with acute cardiovascular events such as stroke and heart attack admitted quickly to local acute care hospitals.

Because of rapid aging and high medical costs for the elderly, the national government amended the Long-term Care Insurance Act in 1997 and the Health Promotion Act in 2002 to improve people's lifestyles in line with the goals set out in Healthy People Japan 2000.

In 2006, the Japanese government amended a reform act regarding the health care system for the prevention and treatment of lifestyle-related diseases to slow down the rise in medical expenditure. The Health Service for the Elderly Act since 1982 has evolved into the Elderly Health Care Security Act, where the health insurer is a central health care provider body rather than municipal governments. Under this Act, the emphasis is placed on the prevention and control of metabolic syndrome where abdominal obesity is an essential component within a constellation of high blood pressure, dyslipidemia, and glucose abnormality. Although persons with metabolic syndrome are at high risk of ischemic stroke and IHD, non-obese persons with high blood pressure, dyslipidemia, or glucose abnormality have a similar or higher prevalence, and are also at high risk.

The threat of increasing incidence of IHD among urban men may justify the recent amendment of the Elderly Health Care Security Act, focusing on preventive and control activities targeting metabolic syndrome. However, it should be noted that the public health burden of cardiovascular disease was equal or even greater among nonobese high-risk individuals compared with obese high-risk individuals. The Elderly Health Care Security Act focusing on metabolic syndrome needs to be re-evaluated and modified in the near future.

AN OVERVIEW OF THE HEALTHCARE SYSTEM IN THE SLOVAK REPUBLIC FOCUSED ON THE INPLEMENTATION OF PREDICTIVE, PREVENTIVE AND PERSONALISED MEDICINE

Kapalla $M^{1,2}$, Kapallová $D^{1,2}$, Turecký $L^{3}$

${ }^{1}$ European Association for Predictive, Preventive and Personalised Medicine (EPMA), Avenue des Volontaire 19, 1160 Brussels, Belgium

${ }^{2}$ Negentropic Systems, Murgašova 12, 03401 Ružomberok, Slovakia

${ }^{3}$ Institute of Medical Chemistry, Biochemistry and Clinical Biochemistry, Faculty of Medicine, Sasinkova 2, Comenius University, 81108 Bratislava, Slovakia 
Healthcare system in the Slovak Republic is neither Bismarck nor Beveridge nor National Health Insurance model, although it has certain features of all. The healthcare contributions are mandatory and are paid to the health insurance company. Almost $100 \%$ solidarity is implemented in the system-people who earn more, pay more, those who earn less pay less, but all receive the same healthcare. There are also healthcare providers who offer their services that are not reimbursed from the health insurance and are paid cash by the patient. Virtually, an insured citizen is not limited as for the amount of the healthcare spending per period of time, on the other hand, the reimbursement limits are set for the particular healthcare providers. According to the data from the health insurance companies, over $98 \%$ of the population is covered by the health insurance. Public healthcare expenditure has been approximately 3400 millions $€$ in 2009 , which represents around $5.36 \%$ of gross domestic product. Another 1000 millon $€$ in cash is estimated to be spent on health-related products and services. In total, around $7 \%$ of gross domestic product is spent in healthcare. On the basis of the statistical data, around $78 \%$ of the 5.4 million population may be regarded to as relatively healthy, or having no apparent health problems. The $22 \%$ of the population suffer of the most common diseases such as cardiovascular diseases, diabetes, respiratory diseases, psychiatric diseases, infectious diseases and cancer. The National Health Promotion Programme states the objectives related to improving the health status in the Slovak Republic. The tools for fulfilling the goals include early prediction, prevention, and education, which is in a good agreement with the concept of predictive, preventive and personalised medicine. The healthcare system in the Slovak Republic has the potential of being substantially better if wisely governed in the years to come (Figure 1). New government has now a unique opportunity to implement the concept of the predictive, preventive and personalised medicine into the healthcare system of the Slovak Republic for the benefit of each citizen and consequently for the entire country. The contacts between EPMA and the Ministry of Health of the Slovak Republic have been established in order to actively initiate the changes needed. The proposed changes include, among other, the following activities:

- more emphasis should be put on the modern laboratory diagnostics and in-vivo diagnostics and their predictive potential

- implement PPPM education programmes at the appropriate levels of education system in the Slovak Republic
- emphasise the role of the healthy nutrition and implement the technology for fast, affordable and convenient analysis of the individual food

- motivate people who do take care of their own health

- stress the personalised aspect of the healthcare, treat the individual patient not the statistical entity called "public health"

- allocate financial resources now and they will return in the lower healthcare spending in the future

- decrease the consumption of medical drugs

- set new standards for quality in healthcare at all levelsprepare the profile of the desired healthcare provider in the particular service (GP, Specialist, Clinical Laboratory, Hospital, Health Insurance, Pharmacy, Emergency medical service, other related services such as education, medical research)

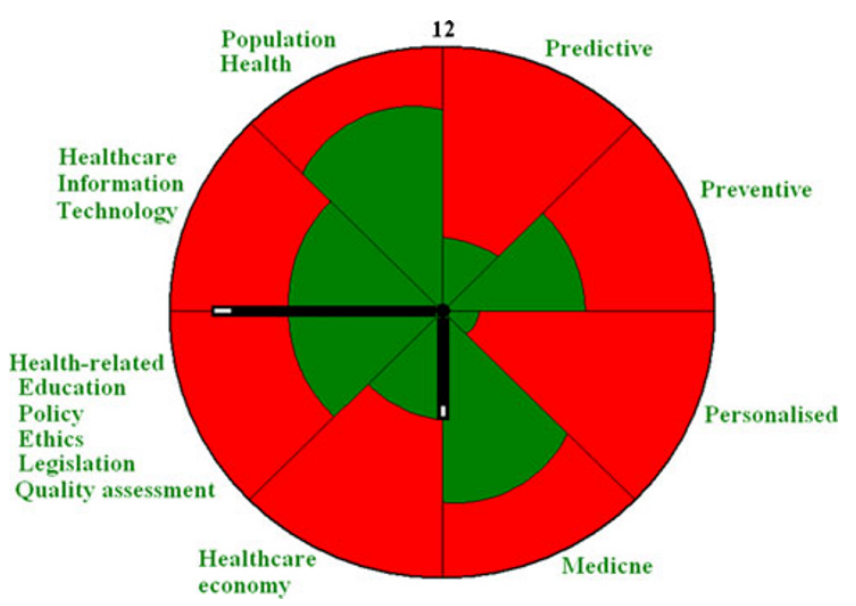

Fig. 1. "Healthcare clock" - a brief visualisation of the healthcare in Slovakia from PPPM implementation point of view. Although particular components may be assessed subjectively to certain extend, authors used verifiable data, information and personal experience for the reliable assessment.

\section{OVERVIEW OF TURKISH HEALTHCARE SYSTEM}

Dundar $M$

Erciyes University Medical Faculty, Department of Medical Genetics, Talas, Kayseri, Turkey

Turkey - one of the most geostrategical countries in the world-is situated at the crossroads of the Old World, Asia, Europe and Africa. It is a major bridge not only between the civilizations of the West and the East but also the main religions followed by billions of people.

Turkey's population recorded as 67.804 .000 in 2000, rose to 73.722 .988 by 2010 . Of this population, males constitute $35.901 .154(50.1 \%)$ and females 35.615 .946 (49.9\%). According figures registered in 2005, life expec- 
tancy is 73.8 years for women and 68.9 years for men (Table 1).

Physicians active in healthcare are 113.151 as registered in 2008. Of these, 57.000 are specialists, 35.000 general practitioners and 20.000 residents in various specialization programs. Other and auxillary medical personnel consists of 20.000 dental surgeons, 100.000 nurses, 78000 medical technicians, 48.000 midwifes, and 25.000 pharmacists.

The public sector continues to be the primary agent for providing healthcare. State owned, public institutions control 92 percent of hospital beds and handle practically all the preventive care services. Meanwhile, private healthcare and medical investments are continually encouraged and on the grow. In addition, as registered in 2008, 830 hemodialysis centers for patients with kidney failure are in service, compared to 443 registered in 2002.

The family physician system, first adopted in 15.09.2005 was enlarged to cover the entire country in 2010. The system allows patients for selectnig their own family physicians and whenever an examination is necessary, they can apply for the doctor of own choice, free of charge excepting actively employed persons, who have to contribute $20 \%$ of the cost of any medication prescribed by the family physician; the retirees' contribution comprises $10 \%$ of the costs. Immunization services are carried out by the family physicians according to a calendar routinely published by the Ministry of Health. Families are not required to make any payment for vaccinations.

At the basic level, Health education in Turkey is provided by mid-level vocational high schools. Then there are vocational colleges attached to universities and at the top, faculties of medicine that raise the physicians. According to the figures of the Higher Education Council, there are 64 Faculties of Medicine in Turkey, 10 in private and 54 in state universities. Health and medical care consequently became more widespread and available to the general public, with such practices as "the green card" as it is popularly called, medical "insurance" for the indigent granted completely free of charge in public institutions.

As for the outreach of medical services, particularly as far as medium and less developed nations are concerned, one most efficient and cost effective method in curbing disease and improving both public and personal health is socializing medical care and spreading preventive medicine to cover larger areas and populations. Turkey's demographic pressures necessitate a comprehensive medical care that requires a larger number of general practitioners but the statistics cited above indicate a serious, if not severe narrowing at the bottom of the pyramid: specialists almost outnumber GPs. Although being free, current healthcare is a subject for further improvements in parallel to essential cultural developments essential to be carry out in the society.

Table1: Demographic Data

\begin{tabular}{llll}
\hline Years & 2000 & 2007 & 2008 \\
Population (x1000) & 67.804 & 70.586 & 71.517 \\
Rural Population (\%) & 35.1 & 29.5 & 25.0 \\
Urban Population (\%) & 64.9 & 70.5 & 75.0 \\
Annual Population Growth Rate (\%) & 13.8 & 11.8 & 11.5 \\
Crude Death Rate (\%) & 6.6 & 6.4 & 6.4 \\
Crude Birth Rate (\%) & 20.3 & 18.1 & 17.9 \\
\hline
\end{tabular}

\section{THE FRENCH HEALTHCARE SYSTEM IN} OVERVIEW

Pinçon-Raymond $M$

93 Route des Gardes, 92190 Meudon, France

The French's healthcare system is totally governed by the law "Bachelot Hôpital, Patients, Santé et Territoires" or law HPST (n² 2009-879 July 21 2009).

The ambition of the law is to reorganize totally the healthcare system: hospital, distribution of general practitioners and private/public access to the cares, public health and prevention, creation of Regional agencies of Health (ARS: Agence Régionale de Santé) in charge of territorially coordination of the health politics (hospital, medicine practices in town, public health and prevention).

The hospital: reorganisation of the assignment and modification of the health institution's statutes.

Distribution: organisation with different levels of care according to the need of the population. The financial participation of the insured patient is required in specific cases.

Public health and prevention: creation of a new agency in charge of the sanitary security, food, environment and work. A particular attention will be made to the side effects of drogues, and therapeutic education of the patients.

ARS: organises the care access and is in charge of the continued health trail of the patient. The ARSs are funded by state contribution, health insurance and local community. The ministry of superior education and health defines the medical formation: territorial regulation of the number of house doctor positions according to the demography and medical specialities needed.

\section{CURRENT HEALTHCARE IN BULGARIA}

Dimitrov $D V^{1}$, Georgieva $T^{2}$, Petrov $K^{2}$

${ }^{1}$ Medical University Varna, Varna, Bulgaria

${ }^{2}$ Health Commission, Bulgarian Parliament 
Since the health system restructuring, Bulgarian health providers are mainly reimbursed prospectively for the services they will provide to the population on a per-case and per-capita basis. This means that actual payment rates are agreed in the contract before the treatment takes place. Each year the National Health Insurance Fund (NHIF), together with the Bulgarian Medical and Dentists Unions, negotiates payment mechanisms to the health facilities contracted with the NHIF. Some types of health provider receive fee-for-service payments, which are a typical form of retrospective reimbursement.

Primary care is provided in private practices, group practices and at outpatient departments. The General Practitioner (GP) is the owner of a private practice and a group practice is owned by its founders. If physicians want to work in the outpatient sector, they are statutorily required to register to provide either primary or outpatient specialized care in either single or group practices; or to be employed by a diagnostic and consultative centre, a medical centre, a dental centre, or a medical and dental centre.

Hospitals receive funding mostly through case-based payments (clinical pathways). An Australian like model, the clinical pathways, was introduced in 2001 as part of the National Framework Contract, based on a single flat rate per pathway. In 2001 there were 158 diagnoses grouped in 30 clinical pathways. Government human resources are assigned to health facilities in accordance with the National Health Map, which specifies by region target numbers of health care professionals per institution. The country will be divided into six regions, each of which will have all types of hospitals. The six regions, which split the country are: North, North Central, North Eastern, South, South Central and Southeast. The aim is that every one of them has all kinds of hospitals-from the GP offices to the most hightech hospitals, so everyone will be able to quickly reach the required medical attention. New unified electronic system for the healthcare is planned to start 2012. It will facilitate communication between different parties (health insurance, hospitals, GPs and patients) and will reduce costs and waiting hours.

The future directions include medical tourism, e.g. for spa and rehabilitation, but also early disease screening enabling personalized medicine that will give advances in public health.

\section{EPMA IN SPAIN: A CHALLENGE FOR FUTURE}

Mendive $J M^{1}$, González Neira $A^{2}$

${ }^{1}$ European Medical Association, Barcelona, Spain

${ }^{2}$ Centro Nacional para la Investigación Oncológica (CNIO), Madrid, Spain

A new structure for the development of the EPMA-related activities is recently being established in Spain. Main aims of the Spanish EPMA network are both to act as national platform for activities on Preventive, Predictive and Personalized Medicine and to provide an international dimension of those activities, acting always on the basis of the international EPMA association and taking into account the importance of providing this international association, an active Spanish network.

To guarantee those aims, Spanish National EPMA Board is being organizing with the presence of relevant people on the on fields of Preventive, Predictive and Personalized Medicine in Spain. The structure of EPMA in Spain is taking into account different institutions, including medical scientific associations with special relevance on research activity as well as medical professional associations and official institutions. Other partners are including possible future private research institutions as partners to guarantee the Spanish EPMA activities. Among these it is being held an active coordination of the advisory board to support a strategic plan of activities that have a promising future of EPMA in Spain.

GEORGIAN HOSPICE MOVEMENT-2000-2011: CHRONOLOGY OF THE KEY EVENTS

Rukhadze T, Velijanashvili M, Alibegashvili T, Kordzaia D Georgian National Association for Palliative Care, Chiaureli st. 0157 Tbilisi, Georgia

During the period 2000-2010 in Georgia, through the permanent collaboration of devotees with Governmental Institutions and NGOs (including International Organizations and Experts) the basis for the development of Palliative Care as an integral part of National Healthcare System was created. It is confirmed by:

- Establishment of "Palliative Care" educational materials in Georgian language, Creation of "Palliative Care" educational programs and their implementation in Medical Universities and Nursing Schools;

- Preparation and implementation of "Palliative Care" CME accredited programs;

- Organization of Hospices (in-patients units for PC) and Home-Based Palliative Care Teams and their financial support by governmental budget;

- Creation of " Palliative Care National Program Coordinator's Office" at the Committee of Labor, Health and Social Affairs of The Parliament of Georgia;

- Preparation of Video/TVand printed materials for public education and awareness;

- Improvement of Legislative-Normative Basis regulating Palliative Care and Drug Availability and promoting Palliative Care incorporation in National Healthcare system of Georgia. 
On April 08, 2007 the amendments in 4 laws of Georgia were approved by Parliament of Georgia:

- Palliative Care was recognized as an integral part of continuous medical aid

- Provision of narcotics and psychotropic substances in required (needed) amount and forms-for medical, scientific and other needs-according to international standards-was recognized as state obligation.

On July 10 of 2008, the Decree of Minister of Labor, Health and Social Affairs of Georgia was issued "On Approval of the Instruction of Palliative Care for Chronic Incurable Patients". This decree includes the following topics:

- Single prescription can cover opioids for 7 days;

- Opioids can be prescribed for chronic or acute pain diagnosis;

- All medical doctors can prescribe opioids after passing special training course;

- Preparation of the medical professionals experienced in PC, including two international fellows (experts).

- Strategy document Georgian National Program for Palliative Care (Action Plan for 2011-2015) was approved by the Parliament of Georgia.

\section{PREVENTIVE, PREDICTIVE, PERSONALIZED AND PARTICIPATORY (P4) HEALTHCARE IMPACTED BY CLIMATE, CULTURE AND COMMUNITY IN THE COMPUTERIZED WORLD}

Gozes $I^{1}$, Marcus-Kalish $M^{2}$

${ }^{1}$ Department of Human Molecular Genetics and Biochemistry, Sackler School of Medicine, Tel Aviv University, Israel

${ }^{2}$ Interdisciplinary Center for Technology Analysis and Forecasting, Tel Aviv University, Tel Aviv 69978, Israel

The goal of Preventive, Predictive, Personalized and Participatory healthcare ("4P") is to lay the strategic refined ground to ushering in a new era of wellbeing and epochal revolution in healthcare to the citizens of Europe and the world. A profound systematic attempt to extract, define, and analyze all relevant factors both inside and outside the body based on the interplay of all scientific disciplines while conveying knowledge tools and expertise. The attempt is to combine the "4P" with the "4C" surrounding factors including Climate, Culture and Community in the Computerized world. Our vision is that the time has come to relate to the individual within the natural and artificial surroundings as one complex functioning system (Figure 1). To further these studies, a special scheme of discussion, will be applied. The CHESTConverging Humanities, Education, Science and Technology methodology will enable placing and tackling the barriers while sharing and converging all concepts, tools and knowhow in a structured well documented procedure.

A systematic analysis including the various treatments and drugs is warranted focusing first on 5 major disease types including brain diseases (e.g. Alzheimer's disease, depression and schizophrenia), metabolic, cardiovascular, cancer and infectious diseases to reduce drug adverse effects. These studies will lay the grounds for better understanding, prevention and therapeutics of other diseases as well. A multilayer system, envisioned in a conceptual matrix approach, will be incorporated to analyze all possible factors in three layers: the healthy population, patients who were diagnosed but not treated and treated patients. The comprehensive analysis in each of these layers (matrix) will try to collect all parameters and effects (rows) in each disease type (columns) while utilizing all available Information Computerized Technologies (ICT) applied and developed in different areas of science.

The deliverables of these innovative cross disciplinary efforts will have a direct impact on:

- Drug efficacy yielding economic impact and reducing world disparities.

- Individual healthcare and wellbeing fitted to the surrounding, with broad impact from economics up to migration and cultural clashes.

- Affordable medicine to the underprivileged population, improving their wellbeing and better utilization of their surrounding will reduce population migration and thus the socio-economic impact.

- Professional and public education utilizing all developed ICT tools, starting from the medical school (thus, reducing animal experimentation) through public education and up to reaching citizen private home providing health care knowledge and support.

- Science research schemes and university structure having to restructure the research management and education schemes towards more open and multidisciplinary implementation.

- Public awareness.

Partners: Prof. Manolis Tsiknakis, Prof. Dr. Michael Hecker, Prof. Marian Joels, Prof. Dr. Bogdan Popescu, Prof. S. Moein Moghimi, Prof. Marianne Schultzberg, Prof. Jan A. Kors , Prof. Olga Golubnitschaja, Dr. Stefan Rüping, Prof. Alfred Nordman, Prof. Milos Kojic, Dr. Enzo Grossi, Prof. Dr. Claus-Michael Lehr, Prof. Jean Eve Blay, Prof. Mauro Ferrari, Dr. Biana Vilentchouk Godin, Prof. Manuela Arata, Dr. Karen Steinmetz, Prof.Seiji Shioda, Prof. Paulo Milani, Dr. Julian Adams, Maurizio Mencarini, Hamutal Meiri, Prof. Ehud Gazit, Prof. Dan Peer, Dr. David Gurewitz, Prof. Eliora Ron, Prof. Mati Mintz, Prof. Yehuda Bnayahu, Dr. Pnina Dan, Dr. Tsipi Shoham, Dr. Zohar Ben Asher 


\section{The Operation flow chart}

\section{Medicine}
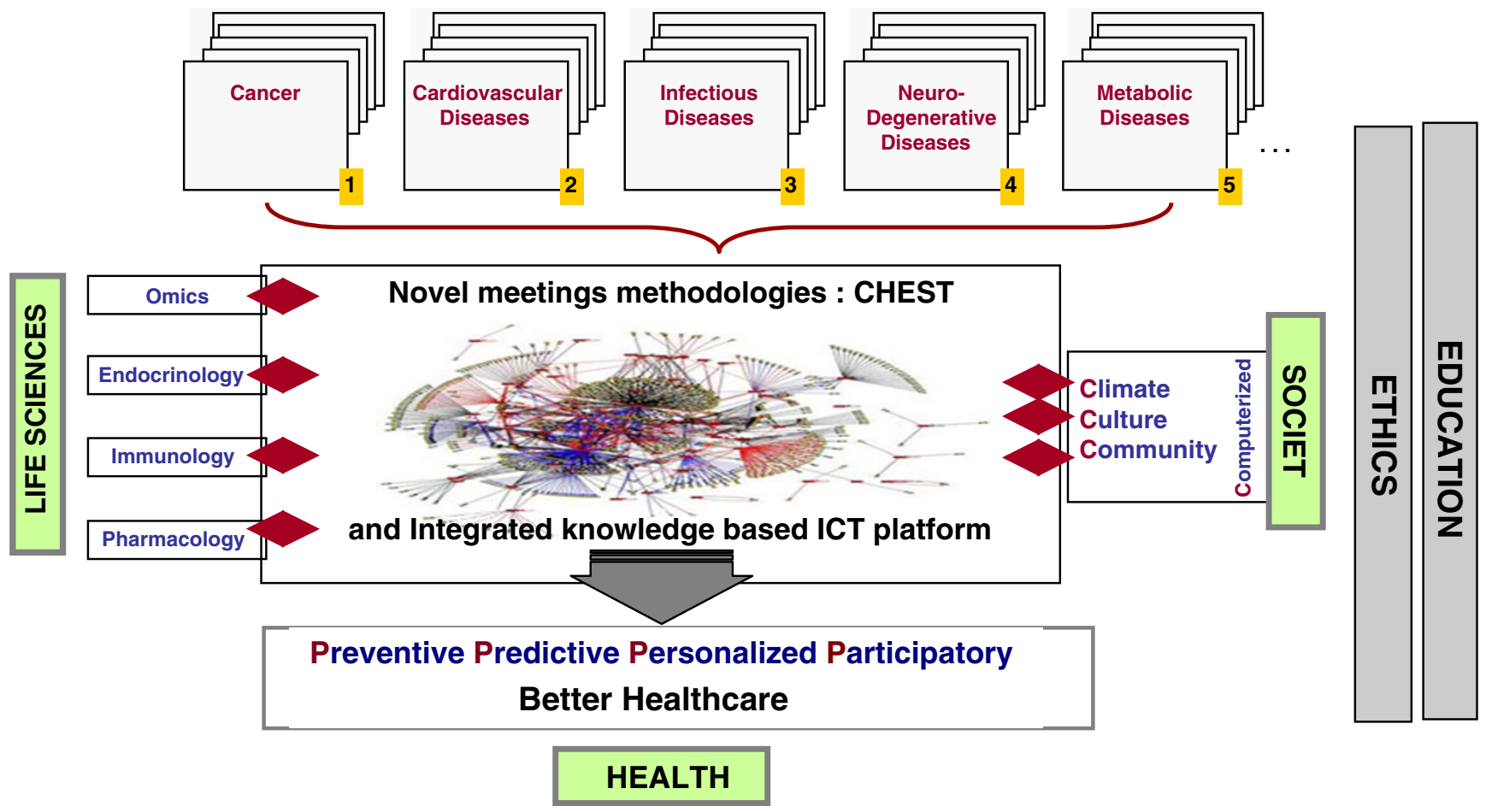

Fig. 1. The Operation flow chart: Multilayer interactions between diverse disiplines will be mediated via a novel superior integrated knowledge based ICT platform and meeting methodologies, e.g. CHEST: The CHEST approach was developed and validate as a novel highly potent methodology tool for $\underline{\text { Converging }} \underline{\text { Humanities, }}$ Education, $\underline{\text { Science \& }}$ Technologies during meetings and workshops discussions, promoting barriers tackling, solution driven discussions and diverse initiatives towards a profound multilevel understanding.

\section{THE ROLE OF MEDICAL IMAGING IN PPPM}

\section{Krestin GP}

Department of Radiology, Erasmus MC, University Medical Center Rotterdam, The Netherlands

"Personalized Medicine" (PM) describes the concept of delivering the right treatment to the right patient at the right time. PM is facilitated by newly developed, powerful technologies allowing detection of biological events at the molecular level, even before symptoms appear. One of the main aspects of PM will be to identify biomarkers able to characterize a cellular alteration leading to subclinical or manifest disease status as well as its specific reaction to various therapeutic attempts. This approach will lead to categorizing or stratifying patients into smaller subgroups related to their risk or potential treatment profiles characterized by a combination of genetic, biochemical, and even imaging biomarkers. However, the value of medical imaging in PM is frequently underestimated, as many policy makers forget the all-important 'right location' in the PM paradigm.

Medical imaging has always been personalized as it provides individual assessment of the location and extent of an abnormality, and in the future it will prove fundamental to almost all aspects of PM. Stratification based on imaging biomarkers can help identify individuals for preventive intervention and can improve disease staging. In vivo visualization of loco-regional physiological, biochemical, and biological processes using molecular imaging can detect diseases in presymptomatic phases or facilitate individualized drug 
delivery. Furthermore, imaging is essential to patienttailored therapy planning, therapy monitoring, and follow-up of disease progression, as well as targeting non-/minimally-invasive treatments, especially with the rise of theranostics. Therefore to reach the highest potential of $\mathrm{PM}$, medical imaging must be an integral part.

\section{INTERNATIONAL CLINICAL RESEARCH INFRA- STRUCTURE NETWORKS TO FACILITATE MULTI- NATIONAL CLINICAL TRIALS AND IMPLEMENT PERSONALISED THERAPIES INTO CLINICAL TRIALS}

Gluud Ch

Copenhagen Trial Unit, Centre for Clinical Intervention Research, Copenhagen University Hospital, Copenhagen, Denmark; Danish National Coordinator, ECRIN

Clinical research plays the key role in the development of health products and medical strategies, leading to evidence-based clinical practice and healthcare cost containment. European Clinical Research Infrastructures Network (ECRIN) is a pan-European infrastructure of national clinical research infrastructures designed to support multinational clinical research, making Europe a single area for clinical trials, taking advantage of its population size to access patients, and unlocking latent scientific potential. ECRIN consists of 14 countries covering more than $80 \%$ of the EU population; nine more countries are expected to join within the next years. ECRIN has been supported by EU FP 6 and FP 7 during 2004 to 2001 (total of Euro 6.8 million). With the support of France, Spain, Italy, and Germany, ECRIN has obtained European Research Infrastructure Consortium (ERIC) status. ECRIN is working for harmonisation of regulatory issues and transparency in clinical research. ECRIN started the servicing of multinational trials in 2010, and more than six trials are presently being conducted. ECRIN plans to expand the network and strengthen the national partners; create a common European culture among professionals and patients communities; support cross-border connection of investigation networks; develop data management and monitoring tools for multinational trials; and make funding available for multinational clinical trials. The expansion includes other world regions, forming the basis for global trials. Such organisation will improve Europe's attractiveness for investigator and industry trials, boost its scientific competitiveness, and result in better healthcare.

Randomised clinical trials are at the top of the evidence hierarchy. The weakness of most randomised trials is that they usually only tell us the outcome of groups of participants randomised to one intervention or the other, giving us a summary of an effect on groups of participants, not information on how the individual responds. Personalised therapies offer what we all request: an in depth, personalised assessment of our heath status and a personally tailored approach to improve or remove any medical problems. If personalised medicine offers 'parachute-like' intervention effects, then randomised trials may not be needed. However, most personalised therapies are likely to offer limited benefit to a restricted group of patients. Such effects are hard to detect. Therefore, personalised medicine must-like other interventions-undergo randomised clinical trials before they can be implemented. We need to demonstrate that the personalised approach is offering more benefits than harms compared with the group-based approach. Therefore, when personalised therapeutic interventions show promise in phase II clinical trials then they should undergo proper assessments in large phase III randomised trials compared with the more simple group-based approach. Only in this way can we assure that the personalised approach is offering the benefits it promises.

ECRIN is poised to offer infrastructures for the conduct of multinational trials on personalised therapies. You just need to contact your national coordinating hub represented by your national ECRIN European Correspondent (www.ECRIN.org).

\section{IMPLEMENTATION OF PERSONALIZED HEALTH- CARE IN HEALTH SERVICES IN THE CONTEXT OF PUBLIC HEALTH GENOMICS-LOOKING FOR THE BROADER PICTURE}

Cesuroglu T, Brand A

Institute for Public Health Genomics, Maastricht University, The Netherlands

The recent developments in basic sciences and health sciences as well as related technologies offer new opportunities for the promotion of population health. This will challenge the healthcare delivery systems in a fundamental way. There is a need for a shift from general recommendations to populations and "one size fits all" strategies towards more personalized interventions in health care. The medicine of the modern world will be Predictive, Personalized, Preemptive/ Preventive, and Participatory, as defined with ' 4 P's of Medicine' or 'P4 Medicine'. Applications of P4 medicine, in particular personalized medicine, combined with the advent of health information technologies in clinical 
practice provide a new kind of medical care: personalized healthcare. It is health care that works better for each individual, based partly on scientific information that is new and partly on technology to make complex information useful.

GENAR Institute for Public Health and Genomics Research, Ankara, Turkey, was established in 2004 as the third public health genomics research centre in Europe. GENAR has developed a personalized healthcare model, called Gentest, based on the use of public health genomics (PHG) tools and concepts to stratify individuals according to risk groups, prevent diseases and detect them early. It has been piloted as a health service to more than 500 individuals since 2008 and was identified as the best practice model for public health genomics in Europe by the Public Health Genomics European Network (PHGEN) in 2008 .

In order to reach the larges coverage and the maximum benefit, the major implementation area of such a practice model is the primary care services in the context of public health. An important part of the European health systems have publicly organized primary care services and the major challenge of implementation of such a model lies in such settings. One of the important problems in the area of implementation of personalized health care is that the concepts, categories, properties, dimensions and relationships forming the ground for implementation of personalized health care in health services, in particular publicly organized primary care is not properly described in the literature. This contribution focuses on the issue.

\section{PREVENTION AND EARLY DIAGNOSIS OF NON- COMMUNICABLE DISEASES THROUGH THE USE OF APPROPRIATE HEALTH TECHNOLOGY}

Maksimovic R, Li Y, Velazquez Berumen A

Diagnostic Imaging and Medical Devices Unit

Essential Health Technologies Department, World Health Organization, 20, via Appia, M13, 1211 Geneva 27, Switzerland

Introduction: The four main noncommunicable diseases (cardiovascular disease, cancers, chronic respiratory diseases and diabetes) lead to an estimated 35 million deaths each year, with $80 \%$ of these deaths occurring in low- and middle- income countries. This number is projected to increase in the future, especially in the African and Eastern Mediterranean regions. Prevention and early diagnosis of these diseases through the use of appropriate health technology can save lives. Policies should exist to ensure equitable access to essential health technologies.
Methods: The $53^{\text {rd }}$ World Health Assembly in May 2000 conceived the Action Plan for the Global Strategy for the Prevention and Control of Noncommunicable Disease. This plan is based on current scientific knowledge, available evidence and a review of international experience. It builds upon the WHO Framework Convention on Tobacco Control and the WHO Global Strategy on Diet, Physical Activity and Health. Building upon this concept, the $60^{\text {th }}$ World Health Assembly passed a resolution on health technologies, recognizing the need to establish priorities in the selection and acquisition of medical devices, and the need to ensure proper use of resources through planning, assessment, acquisition and management. In April 2011, the First Global Ministerial Conference on Healthy lifestyles and noncommunicable disease control met with an agenda to push forward equitable access to health technology.

Results: The Action Plan for Noncommunicable Diseases targets the four main noncommunicable diseases through control of four shared risk factors: tobacco use, physical inactivity, unhealthy diets and harmful use of alcohol. The objectives of the action plan are to: 1. Raise awareness of the priority of noncommunicable disease in development work at the global and national levels, 2. To establish and strengthen national policies for prevention and control of noncommunicable diseases, 3. To promote interventions to reduce the main shared modified risks factors for noncommunicable diseases, 4. To promote research for the prevention and control of noncommunicable diseases, 5. To promote partnerships for the prevention and control of noncommunicable diseases, and 6. To monitor noncommunicable diseases and their determinants. For each objective, the Action Plan outlines proposed actions for the Secretariat, Member States, as well as International Partners. The role of health technologies in achieving these objectives is outlined in the $60^{\text {th }}$ World Health Assembly health technologies resolution. Countries are urged to collect, verify, update and exchange information on medical devices and to formulate appropriate national strategies. They are urged to draw up national and regional guidelines for good manufacturing and regulatory practices, to establish the necessary collaborations and centers of technology, and to collect information that inter-relates medical devices with public health infrastructure.

Conclusion: Low- and middle-income countries share a majority of the burden of noncommunicable disease, and the incidence continues to rise disproportionately in these countries. The WHO seeks to raise the awareness of the use of appropriate, affordable technology, particularly diagnostic imaging technology, for the prevention and early diagnosis of noncommunicable disease. 

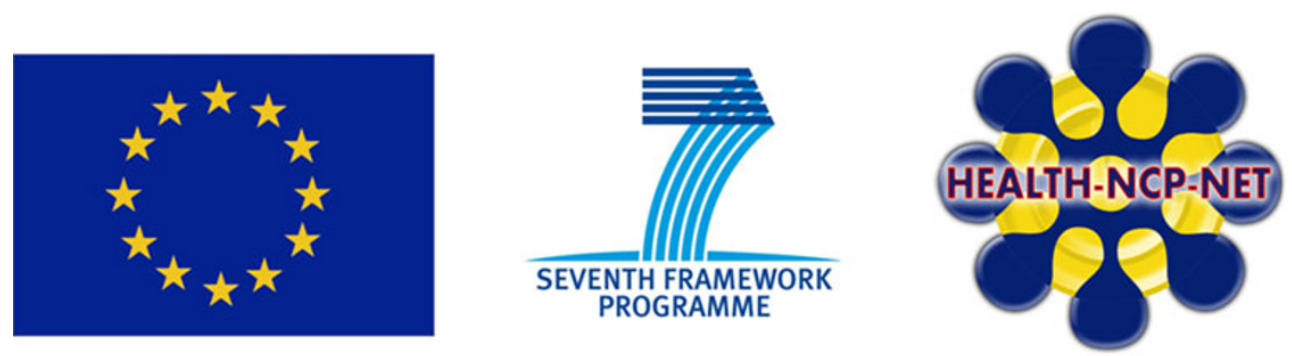

\section{Health - NCP - Net \\ www.healthncpnet.eu \\ Your Support Network}

\section{HEALTH-NCP-NET \\ Kinkorová $J$ \\ Health NCP of the Czech Republic}

This project is a Coordination Action for Reinforcing the Health National Contact Points Network in the $7^{\text {th }}$ Framework Programme for Research and Development, 2007-2013. It has been running since May 2008 and will end in April 2012. This project has received a total funding of XXX euros. Health-NCP-Net is the network of officially appointed Health National Contact Points (NCPs) and Contact Points in International Cooperation Partner Countries (ICPC CPs). THe consortium consists of 19 partners; the coordinator is Instituto De Salud Carlos III, ISCIII, Spain.

The objective of HEALTH-NCP-NET is to improve NCPs' services under FP7 across Europe, as well as in ICPC countries by providing a more consistent NCP support that will finally result in a higher quality of submitted proposals into topics for the Health priority by applicants. The aim is to develop a standard method of work, whilst keeping in mind that diversity exists between NCP systems per country; these can vary in structure, personnel, funding schemes and advising capacity.

The mission of the project is to provide all Health NCPs and ICPC CPs with the necessary tools, guidelines, advice and support to carry out their duty efficiently and, most importantly, to be part of a dynamic network. Health-NCPNet offers training sessions and encourages the exchange of experiences and best practices amongst NCPs enabling them to learn from each other's experience.

The activities in the context of the Health-NCP-Net project are mainly benchmarking, networking, transnational brokerage events and the training programs. It is in the spirit of the project partners to share the existing know-how and to utilize already existing resources.

Attention is given to less experienced NCPs as well as to Contact Points in ICPCs, so that they rapidly acquire the knowhow accumulated in other countries to perform their work efficiently. An effort is devoted to identify and involve in the network all official FP7 contact points from the ICPCs and to promote the enlarging of the network by setting up new contact points for Health in countries where these are not yet present.

\section{SALUTOGENESIS AND AYURVEDA: INDICATIONS FOR PUBLIC HEALTH MANAGEMENT}

Morandi $A^{1,2}$, Dalla Libera $D^{1,2,3}$

${ }^{1}$ Ayurvedic Point, C.so Sempione 63, 20149 Milan, Italy

${ }^{2}$ SSIMA, Italian Scientific Society for Ayurvedic Medicine, C.so Sempione 63, 20149 Milan, Italy

${ }^{3}$ IRCCS San Raffaele, DIMER, Dept of Neurology, Via Olgettina 48, 20132 Milan, Italy

Ayurveda (from Sanskrit "Science of Life") - the ancient Traditional medicine native to India- describes health as "stability in the true self" (swastha), a state of complete, balanced, physical, mental and spiritual well-being [1]. This remind us to United Nations' World Health Organization's definition of health as "a state of complete physical, mental, and social well-being and not merely the absence of disease or infirmity" [2].

In Ayurveda the focus is about the person who has a disease not about disease itself and its concerns are maintenance of an abiding health, preventing rather than treating diseases. This is in accordance to what Winslow claimed in 1920 defining public health as "the science and art of preventing disease, prolonging life and promoting health through the organized efforts and informed choices of society, public and private organizations, communities and individuals" [3].

Because of the sharp increase in non-communicable disease $[4,5]$-especially on developing countries-public health has to find strategies for prevention and control of chronic diseases -issue of 2011 UN General Assembly- through surveillance of cases and promotion of healthy behaviours. Ayurveda propose a low-cost-regimen of personalized healthy conduct according to the individual (swasthavritta) involving lifestyle, nutrition, self-awareness and good 
relationships with other people and nature [6]. It is necessary to build a framework that support patients' and family interactions at the micro-level and a wellcoordinated policy and health-care systems, with attention to social and environmental aspects [7]. In fact, relationships between individuals in turn define individual themselves, contributing to awareness and self-realization and consequently, to the sense of well-being within communities. Another aim is to develop meaningful relationships between community members and public health practitioners. As stated by Charaka Samhita Sutrasthana 9: 26 - one of the main Ayurvedic textbook- " a doctor should be sympathetic and kind to all patients", he must consider each individual's perception of the world, inner "sense of coherence" and mindbody interactions. Communication should be improved and patient involvement enhanced, so that people are made responsible for their health.

Public Health Management needs to promote Health education-as announced by the 2012 WHO health report "stimulating the generation, translation and dissemination of valuable knowledge"- since early ages and integrate spirituality and ethics in healthcare system. A multidisciplinary professional teams may be required, including physicians specialized in public health, nutritionists, physiotherapists, psychologists, social scientists, biostatisticians, environmental health officers, public health engineers and lawyers, sociologists, communications officers and others [8].

Many diseases may be preventable through simple, nonmedical methods (diet, meditation, massage) since infancy, in order to improve life quality, limit drugs use (avoiding expensive side-effects) and reduce medical costs.

Public Health need to address health inequalities by advocating for population-based policies that improve health in an equitable manner. A proposal comes from traditional medicines, both in developing countries-where they are affordable, available, and culturally familiar- and in industrialized countries- since their integration in healthcare in a more holistic approach may help disease management $[9,10]$. Ayurveda, being person-centered is intercultural, meeting the needs of different populations thanks to the universal-coverage in its roots.

\section{References:}

1. Sharma H, Chandola HM, Singh G, Basisht G. Utilization of Ayurveda in healthcare: an approach for prevention, health promotion, and treatment of disease. Part 2-Ayurveda in primary health care. J Altern Complement Med. 2007;13:1135-50.

2. Preamble to the Constitution of the World Health Organization as adopted by the International Health Conference, New York, 19-22 June, 1946
3. Jekel JF. Health departments in the U.S. 1920-1988: statements of mission with special reference to the role of C.-E.A. Winslow. Yale J Biol Med. 1991;64:467-79.

4. Roberti di Sarsina P. The Social Demand for a Medicine Focused on the Person: The Contribution of CAM to Healthcare and Healthgenesis. Evid Based Complement Alternat Med. 2007;4 (Suppl 1):45-51.

5. Allotey P, Reidpath DD, Yasin S, Chan CK, de-Graft Aikins A. Rethinking health-care systems: a focus on chronicity. Lancet. 2011;377(9764):450-1.

6. Geneau R, Stuckler D, Stachenko S, McKee M, Ebrahim S, Basu S, et al. Raising the priority of preventing chronic diseases: a political process. Lancet. 2010 Nov 13;376(9753):1689-98.

7. Schultz AM, Chao SM, McGinnis JM. Integrative Medicine and the Health of the Public: A Summary of the February 2009 Summit. 2009. http://www.equilibriume3.com/images/PDF/2009\% 20National $\% 20$ Summit $\% 20$ on $\% 20$ Integrative $\% 20$ Medicine.pdf

8. Burr W. WHO moves to classify traditional medicines. CMAJ. 2011;183(2):E73-4.

9. Sengupta A. Towards a truly universal Indian health system. Lancet. 2011;377(9767):702-3.

10. Alves RR, Rosa IM Biodiversity, traditional medicine and public health: where do they meet? J Ethnobiol Ethnomed. 2007;3:14.

\section{INCORPORATION OF NATIONAL CANCER CON- TROL PLANE IN GEORGIAN HEALTH CARE SYSTEM}

Maglakelidze M, Jokhadze N, Rukhadze T, Gagua R

National Cancer Center of Georgia, Association of Oncologists of Georgia, Lisi Lake, 0177 Tbilisi, Georgia

Background: Over 7.000 people are diagnosed with cancer in Georgia annually. Every year nearly 4.500 patients die from cancer. Cancer is a second leading cause of death and main priority in Georgian Health Care System.

Unfortunately, $70-72 \%$ of newly revealed cancer cases are diagnosed at stage III-IV. More than half are resistant to treatment and rest require long term, costly treatment, medical, social and psychological rehabilitation. Many cancer patients became disabled, abandon active lifestyle and often need a caregiver, what constitutes a heavy burden for their families and the public.

Objectives: The purpose was to create and present to government Georgian National Cancer Control Plan (NCCP). Cancer Control aims to reduce burden and suffering from cancer by preventing exposure to risk factors, early detection, effective treatment, pain and symptom management of incurable patients.

Combination of different approaches involving education, awareness, advocacy, legislation, vaccination, screening, early detection and treatment is used to prevent cancer incidence and mortality. The NCCP objectives for reducing the cancer burden in Georgia 
are based on Surveillance data and the national objectives from Cancer Registry.

Methods: The cancer professional community and policymakers spent a lot of time developing a comprehensive cancer control plan. Between September and October 2010 there was an open public debate. Later, during November 2010 additional consultations on the Plan took place. In 2011 after permanent public debates and consultations final version was adopted by Health Council. This enables to enact certain programs and activities already in 2011. With input from more than 20 professional, academic, and community-based organizations that worked together 10 major goals were identified in the field of cancer control.

\section{NCCP objectives:}

- Promoting cancer data collection.

- Rise in public cancer awareness and healthy lifestyle popularization.

- Enhancement of healthy behaviors.

- Increasing screening rates

- Improving access to the full spectrum of cancer diagnosis and care.

- Implementation of National Diagnostic and Treatment Guidelines.

- Increasing patient, caregiver, health care professional awareness about issues of quality of life, increase in access to palliation and pain control.

- Improve reporting quality of cancer incidence, mortality, and staging data.

- Improvement of oncology service at each level.

NCCP Goals:

- Primary Prevention;

- Early Detection;

- Treatment and Cancer Care;

- Research and Clinical Trials;

- Palliation and Quality of Life;

- Survivorship;

- Data and Surveillance.

Results: NCCP sets out comprehensive strategy in fight against cancer. It's the first time government adopted major program of action linking prevention, diagnosis, treatment, care and research. As a result several governmental programs have been founded.

Conclusion: Although the reasons for declining cancer rates are multifaceted, the efforts of the NCC play an integral part. NCCP aims to reduce cancer mortality and to influence selected behaviors that influence cancer mortality. This new set of goals, objectives, and strategies is intended as a framework for continued improvements by Year 2020.
TRADITIONAL AND NON-CONVENTIONAL MEDICINES: THE SOCIO-ANTHROPOLOGICAL AND BIOETHICAL PARADIGMS FOR PERSONCENTRED MEDICINE, THE ITALIAN CONTEXT

Roberti di Sarsina $P^{l}$, Iseppato $I^{2}$

${ }^{1}$ High Council of Health, Ministry of Health, Italy; Observatory and Methods for Health, Department of Sociology and Social Research, University of Milan-Bicocca, Italy

${ }^{2}$ University of Bologna, Italy

Abstract: In Italy the use of non-conventional medicines (NCM) is spreading among people as in the rest of Europe. However, in Italy, unlike that in other countries of the European Union, at the present time the juridical/legal status of NCM is not well established, mainly due to the lack of any national law regulating NCM professional training, practice and public supply and to the absence of government-promoted scientific research in this field. This is an obstacle to safeguarding the patient's interests and freedom of choice, especially now that dissatisfaction with biomedicine is inclining more and more people to look for a holistic, fairer and patient-centred form of medicine.

Safeguarding people's health, making treatment personal, human and sustainable, presupposes that the patient be central in choosing the therapy. Society has changed its perception of quality in medicine and is demanding that institutions humanize medicine: it must be both humanistic and scientifically-based, hence person-centred medicine. Diagnosing and treating each human being requires that we find an individual centre of gravity taking the whole of the person into consideration, their intrinsic unity, physical and mental: the incessant interaction of these levels forms the spiritual core of the human being, a unicum to be interpreted before it can be cured.

There is an evident gap between a classic "healthcare system" and a "pro-health system" of the kind needed to reprogramme public health in the teeth of worldwide economic crisis. The traditional health system does not cover the full implications of health: there are so many significant factors that must be embraced by a pro-health system. It is imperative that we integrate and liaise among the various approaches to medicine as an art; a synergy must be struck between biomedicine and anthropological or traditional and non-conventional medicines, if we are to achieve a sustainable equilibrium and contain the cost of drugs.

It is no longer enough to learn about the origins of pathology; traditional and non-conventional medicines share the commitment to broadcast knowledge of how to generate health, entailing studies on the sources of physical, psychological and spiritual health; they share the ethical and social mission to generate greater responsibility and social emancipation. 
Science stands in need of new paradigms of pluralism. That challenge for the future extends beyond biomedicine: we need to review the evidence-based approach and apply it to traditional and non-conventional medicine. Not only are the public health authorities still reticent about traditional and non-conventional medicine, the whole health system needs to set in motion a virtuous process whose central aim should be global care of the individual patientperson-centred medicine - a caring diachronic approach to the psycho-patho-biological human being. Ethics demand that we preserve, protect, promote, study, hand on and apply the cultural heritage of medical and anthropological health expertise, be it western or eastern, respecting the original integrity of the tradition paradigms and patrimonies. The epistemological status of medicine calls for reformulation, and the key to hand is "person-centred medicine".

\section{TIBETAN MEDICINE: A UNIQUE HERITAGE OF PERSONALIZED MEDICINE}

Roberti di Sarsina $P^{l}$, Ottaviani $L^{2}$

${ }^{1}$ High Council of Health, Ministry of Health, Italy; Observatory and Methods for Health, Universtity of Milano-Bicocca, Italy

${ }^{2}$ International Shang Shung Institute for Tibetan Studies, Italy

With a history going back approximately 2500 years, the Tibetan Medicine, known as Sowa Rigpa in the Tibetan language, is one of the world's oldest known Traditional Medicine. It originally developed during the pre-Buddhist era in the kingdom known as Shang Shung.

Over time it integrated religious influences and became the basis of healing science in Tibet. In the 7th and 8th century the Kings of Tibet promoted the exchange of healing knowledge from outside the kingdom, and physicians from India, Nepal, Persia and China came to Tibet to discuss their medical systems. The result is believed to be a collection, in Tibetan, of their combined medical knowledge, formalized in the Four Tantras by Yutok Yonten Gonpo.

The fundamental theory of Tibetan Medicine sees a healthy body composed of three humors, seven basic constituents and three excretory substances. An unbalance in these components then causes disease. Diagnosis is performed through observation, palpation, questioning, urine, pulse, and tongue analysis. It's very interesting to point that treatments not only cover the use of medicines, but also diet and behavior along with physical therapies. Traditional Tibetan Medicine is an ancient science in which some of its fundamental principles are listening carefully to the patient, a close global examination (body, mind, energy, but also surrounding circumstances) and personalized medical care.

Due to its cultural roots and to the approach based on the specific identity of the patient in relation to his whole environment (life history, personal issues, context and feelings), Tibetan Medicine can be seen as a perfect example of Person-Centred Medicine. In particular it enhances the relationship between patient, doctor, and society as an important aspect of health. The disease is considered in its full dimensions with a primary focus on general mental health and collaboration with one's culture. In addition daily integration of diet, prescribing particular foods to particular people, is an effective method of preventive health as well as for the treatment of mild disorders.

The Tibetan Medical Tradition has continued to grow into modernity. After the Chinese invasion of Tibet, in 1961 His Holiness the 14th Dalai Lama in exile re-established (original created in Lhasa, 1916) the Men-Tsee-Khang, Tibetan Medical and Astrology Institute, in Dharamsala, India. Doctors there and abroad have had increases success developing research and treatment for many illnesses, especially those that have become chronic to which there is no clear biomedical cures.

At present in China where Traditional Chinese Medicine is not only well-developed but also part of the dominant health system, Traditional Tibetan Medicine has been accepted within the medical system as an autonomous Traditional Medicine not being confused with TCM, and it is slowly growing in popularity. Western countries have only recently gained access to the Tibetan Buddhist texts which provide some of the theoretical framework for Tibetan medicine, with yet too few translations of the main texts (including the four Tantras). At the same time schools of Traditional Tibetan Medicine, recognized by the Tibetan Medical and Astrology Institute, are slowly being created and growing in US and Europe providing a useful source of knowledge to both doctors willing to integrate them in Western practice and patients seeking alternative healing modalities.

As a Traditional Medicine, the future development of Tibetan Medicine in Western countries is linked to being recognized as popular and viable health care option providing an alternative clinical reality. At the same time it is invaluable to preserve its expression as both a culturally centered and holistic patient based traditional medicine.

On Semptember 11th 2010 the ancient Tibetan medical system was the core of the keynote lecture given by prof. Chögyal Namkhai Norbu Rinpoche in the main hall of the Institute of Anatomy of Bologna University.

Prof. Namkhai Norbu, one of the most important living Masters of the Tibetan Tradition and Holder of the Dzogchen Lineage, delivered a keynote address entitled "Tibetan 
Medicine, Heritage of the Mankind" in the Auditorium of the Institute of Anatomy of the University of Bologna.

This was the first time that the Auditorium of the Institute of Anatomy at Bologna University has been open for a public address. http://www.dzogchen.it/press/?news_ it\&n=1288540491 620

The event which was organized by the Associazione per la Medicina Centrata sulla Persona Onlus (Association for Person Centered Medicine, Onlus, a Charity established according to the Italian Law) in collaboration with the International Shang Shung Institute for Tibetan Studies, in acknowledgement of its overall significance, has been granted of highest level endorsements, among which The European Association for Predictive, Preventive and Personalised Medicine and The European Medical Association. http://www.dzogchen.it/lp/conferenza_2010-09-11/ index.php?lang=en

The International Shang Shung Institute was founded in Italy in 1989 by Chögyal Namkhai Norbu Rinpoche, and inaugurated in 1990 by His Holiness the 14th Dalai Lama. It's aim is to deepen the knowledge and understanding of Tibetan cultural traditions in order to ensure their survival and preservation. The International Institute currently has three main branches, located in Italy, Austria, and the USA, which work together as one to promote all initiatives to this end. The mission of the Shang Shung Institute is to deepen the knowledge and the understanding of the Tibetan cultural traditions in its religious, historical, philosophical, artistic, medical, and social aspects in order to contribute to the survival and preservation of this culture. Its purpose is to encourage cooperation among interested groups and individuals, thus keeping alive the essential values of these traditions. "The Shang Shung Institute exists for the preservation of Tibetan Culture and to translate Tibetan books into Western languages. Through the Shang Shung Institute we are trying to do something to maintain the survival of Tibetan Culture and understanding. Shang Shung, the name of the Institute, reflects the source of Tibetan culture and history-it was known as a great empire throughout the Orient and the study of Shang Shung is extremely important if we are to understand the great antiquity, the unique nature and the universal importance of Tibetan Culture, past and present." Prof. Chögyal Namkhai Norbu Rinpoche, Founder of the Shang Shung Institute

\section{References:}

1. Men-Tsee-Khang Tibetan Medical \& Astrological Institute of H.H. The Dalai Lama

http://www.men-tsee-khang.org
2. The International Shang Shung Institute for Tibetan Studies http://www.shangshunginstitute.org

3. The Shang Shung Tibetan Healing Center

http://www.tibetanmedicinecenter.com/shangshung/Welcome.html

4. The International Shang Shung Institute for Tibetan StudiesMultimedia Portal

http://www.shangshunginstitute.com/index.php

5. The Shang Shung School of Tibetan Medicine

http://shangshung.org/home/school.html

6. Associazione per la Medicina Centrata sulla Persona Onlus-Charity Association for Person Centered Medicine, Bologna, Italy

http://www.medicinacentratasullapersona.org

7. Merigar-International Dzogchen Community

http://www.dzogchen.it

TOWARDS SALUTOGENESIS IN THE DEVELOPMENT OF A PERSONALISED AND PREVENTATIVE HEALTHCARE

Alivia $M^{1,2}$, Guadagni $P^{2}$, Roberti di Sarsina $P^{2,3}$

${ }^{1}$ Italian Society of Antroposophic Medicine (SIMA)

${ }^{2}$ Charity "Association for Person Centred Medicine", Bologna, Italy

${ }^{3}$ High Council of Health, Ministry of Health, Italy

The purpose of this review is to discuss how a salutogenetic approach may solve some of the difficulties faced by healthcare systems. Salutogenesis explores the reasons why some people stay healthy in the face of hazardous influences whilst others, faced with the same hardship, fall ill. It was Antonovsky who suggested that resilience to difficult situations depends on a person's sense of coherence (SOC), a global orientation based on a sense of self-reliance in the face of challenges, self-confidence in one's ability to deal with demanding events and the motivation to 'keep going', trusting that such difficult events hold meaning for one's life $[1,2]$. There is a large body of research on all age groups, different socioeconomic backgrounds and across cultures that shows that a strong SOC is related to better health and healthy ageing. It is a protective factor against alcohol addiction despite similar rates of recreational consumption in teenagers. Conversely, a weak SOC is related to poorer health and lower affect. SOC is a dynamic orientation that develops naturally in the first 30 years of life but can also be strengthened through personal activity and care [3-6].

How can a healthcare system be oriented so that overcoming illness becomes the foundation of better future health? In such a system each medical intervention should aim to strengthen a person's SOC and resilience and this should be differentiated at each level from primary to tertiary care. Each professional role too should be developed to 
provide salutogenetic care from their angle and this should be addressed from as early as undergraduate training [7]. They should work towards the principles of personcentred-medicine, a concept that aims to incorporate the holistic approach, the relationship-based care and the treatment systems of non-conventional medicine into the technological advances of mainstream medicine in order to provide more personalised and sustainable healthcare $[8,9]$.

The caregiver-person relationship needs to become central to the therapeutic process so that any treatment is chosen as an informed choice, based on the needs of that person in the context of their physical, psychological and biographical situation. As an example, if the chosen treatment were a coronary artery bypass graft then the technological aspects would need to be chosen specifically for that single case. However for the whole process from pre-operative care to rehabilitation to become a salutogenetic experience, physical as well as psychological, social and personal factors need to be addressed at the appropriate time. This could develop the awareness that overcoming this difficult experience was meaningful because it allowed the development of new life skills, new coping strategies, and new confidence in one's healing abilities [10]. This change needs to be sustained over time with appropriate follow-up [11].

Adopting a healthier lifestyle is likely to improve the quality of life of that person, and may prevent future illness being cheaper in the long run for the healthcare system. In order to evaluate the success of medical interventions broadened with a salutogenetic approach, parameters may need to be developed and adopted that can assess quality of life of patients and caregivers in terms of sustainable change, satisfaction and prevention of burn-out as well as cost-effectiveness and technological excellence [12].

\section{References}

1. Antonovsky A. Unravelling the mystery of health: how people manage stress and stay well. 1st edition. San Francisco: JosseyBass; 1987

2. Johnson M. Approaching the salutogenesis of sense of coherence: The role of 'active' self-esteem and coping. Br J Health Psychol. 2004;9:419-432

3. Fagerstrom L, Gustafson Y, Jakobsson G, Johansson S, Vartiainen P. Sense of security amongst people aged 65 and 75: external and inner sources of security. J Adv Nurs. 2011; doi:10.1111/j.13652648.2010.05562.x
4. Lee YY, Chan MF, Mok E. Effectiveness of Music Intervention on the quality of life of older people. J Adv Nurs. 2010;66(12):267787.

5. Lutgendorf S, Vitaliano P, Tripp-Reimer T, Harvey JH, Lubaroff DM. Sense of coherence moderates the relationship between life-stress and natural killer cell activity in healthy older adults. Psychol Aging. 1999; 14:552-63.

6. Bäärnhielm S. Restructuring Illness Meaning Through The Clinical Encounter: A Process Of Disruption And Coherence. Cult Med Psychiatry. 2004;28:41-65

7. Rakel DP, Guerrera MP, Bayles BP, Desai GJ, Ferrara E. CAM Education: Promoting a Salutogenic Focus in Health Care. J Altern Complement Med. 2008;14(1):87-93.

8. Roberti di Sarsina P, Iseppato I. Person-Centred Medicine: Towards a Definition. Forsch Komplementmed. 2010;17:277-8.

9. Roberti di Sarsina P. The Social Demand for a Medicine Focused on the Person: The Contribution of CAM to Healthcare and Healthgenesis. eCAM. 2007;4(S1)45-51.

10. Karlsson I, Berglin E, Larsson PA. Sense of Coherence: Quality of Life before and after Coronary Artery bypass Surgery: A longitudinal study. J Adv Nurs. 2000;31(6):1383-92.

11. Giallauria F, Battimiello V, Veneziano M, De Luca P, Cipollaro I, Buonincontro M, Vigorito C, Del Forno D. Psychosocial risk factors in cardiac practice. Monaldi Arch Chest Dis. 2007;68:74 80

12. Ventegodt S, Omar HA, Merrick J. Quality of Life as Medicine: Interventions that Induce Salutogenesis. A Review of the Literature. Soc Indic Res. 2011; doi:10.1007/s11205-010-9621-8

\section{HELP4MOOD PROJECT: TECHNOLOGY TO HELP IN PERSONALIZED MANAGEMENT OF DEPRESSION}

Mendive $J^{1,2}$, Fernández $A^{2}$, Rubio $M^{2}$, Luciano $\mathrm{JV}^{2}$, Baladón $L^{2}$, Barreda $V^{2}$, Serrano-Blanco $A^{2}$, on behalf of the Help4Mood Partnership

${ }^{1}$ Barcelona, Spain, EPMA Spanish National Representative ${ }^{2}$ Health Technology and Outcomes in Primary Care and Mental Health Group, Research and development unit, Parc Sanitari Sant Joan de Déu Sant Boi de Llobregat, Barcelona, Spain

The aim of the Help4Mood project is to develop a system that will help people with major depression recover in their own home. The Help4Mood system is designed to be used together with other forms of therapy, such as self-help, counselling, or medication. Help4Mood has three main components:

- a personal monitoring system that keeps track of important aspects of behaviour such as sleep or activity levels

- an interactive virtual agent who asks patients about their health and well-being, provides a portal to trusted health 
information, and feeds back information collected through monitoring and questionnaires

- a decision support system that tailors each session with the virtual agent to the individual needs of the person with depression and that supports clinicians in interpreting the data collected through the virtual agent and the personal monitoring system.
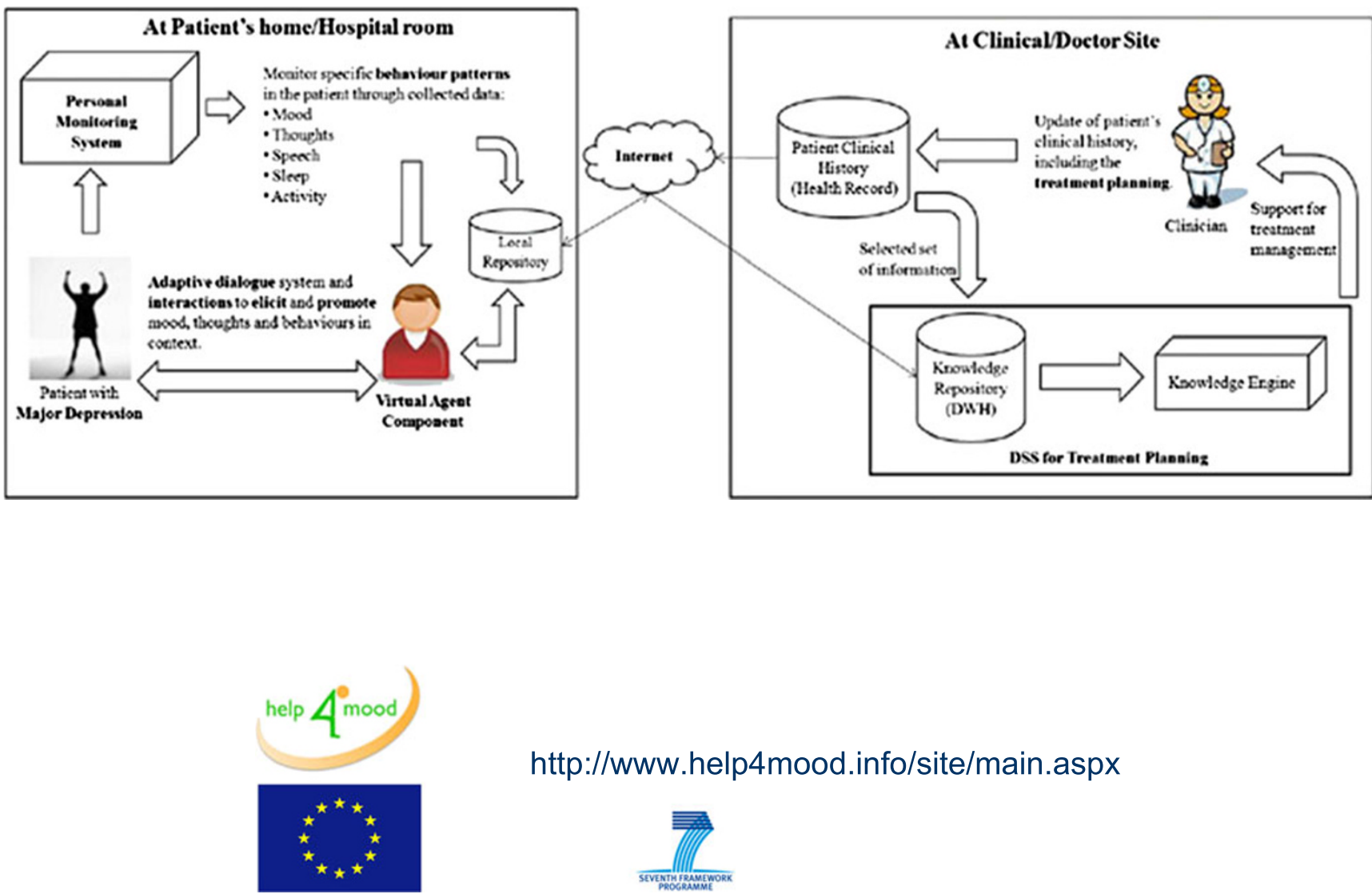

http://www.help4mood.info/site/main.aspx

EU Funded Project on the Seventh Framework Programme

Partners: of EDINBURGH

Medical
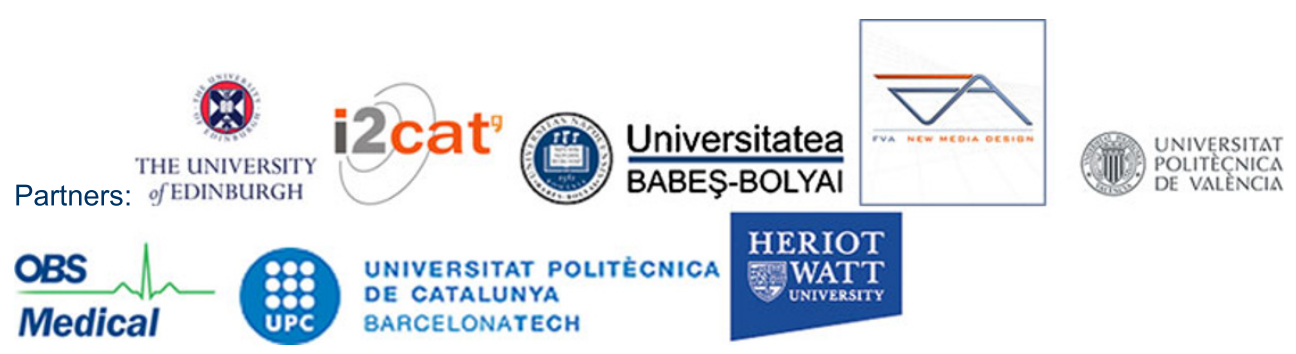

UNIVERSITAT POLITÉCNICA

DE CATALUNYA

BARCELONATECH

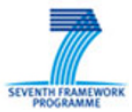

S for Treatment Planning 


\section{PERSONALITY'S PROFILES OF TEENAGERS AND STUDENTS: A COMPARATIVE STUDY PERFORMED IN TIMISOARA, ROMANIA}

\section{Petrescu C, Suciu O, Ursoniu S, Vlaicu B}

"Victor Babes" University of Medicine and Pharmacy, Timisoara, Romania

Personality's profilles are established individually. Observation of a large group of persons with the same age offers the possibility to establish what personality features are pregnant for a specific stage of human development.

The aim of the study performed was to investigate the features of the personality considering age group peculiarities. The measurable objectives were: to realize individual profiles of personalities and group profiles with relevance of the pregnant features on age group; to relate pregnant features of an age group with risk behaviors; to compare the age group peculiarities in relation to risk behaviors between teenagers and students.

The materials consisted of two samples: a sample of teenagers (2908, 1498 girls and 1410 boys) and a sample of students (2124, 1074 girls and 638 boys). There was a problem with the second sample, were girls were more numerous than boys. We used the method of an epidemiological inquiry based on two questionnaires: CORT 2004 (with 126 items that investigated smoking, alcohol and drug consumption, aggressive behaviors, traffic accidents, sexual, feeding, sedentary and self-medication behaviors) and Freiburg Personality Inventory (FPI) with 212 items that investigated nervousness, aggressiveness, depression, excitability, sociability, calm, domination, inhibition, sincerity, emotional lability, bipolar personality features. Both questionnaires were individually applied, in one session and respecting anonymity.

Results obtained: Four personality's dimensions presented ranks over 6 (4- 6 being considered balanced) in teenagers: nervousness (rank 8), aggressiveness, excitability and domination (rank 7 each of them). Two personality's dimensions were registered with high ranks in students: nervousness and sincerity (rank 7, each of them). Risk behaviors related with personality features in teenagers are: smoking and alcohol consumption-the same profiles with the sample; drug consumption-oscillation on the depression dimension; hetero-aggressive behavior-increased ranks of nervousness and aggressiveness; suicidal behavior-increased level of depression and history of suicide in family and also increased ranks of nervousness and aggressiveness, sexual behavior with risk-decreased domination, decreased sociability and aggressiveness. There was a tendency in teenagers to lie and exaggerate the number of sexual partners. Risk behaviors related with personality features in students are: smoking - the same profile with the samples, alcohol and drug consumption-decreased level of inhibition; hetero-aggressive behavior-oscillation of the sociability dimension (introvert-physical aggressiveness, extrovert-verbal aggressiveness), suicidal behaviors-no pregnancy of features; sexual behavior with risk-high level of sociability and of sincerity dimensions.

In conclusion, there are relevant differences between teenagers and students as far as personality features (students are more balanced than teenagers) and risk behaviors are concerned (in teenagers they are connected with instability and in students they are connected with specific dimensions of personality and they seem to be more persistent).

\section{EVALUATION OF THE IMMUNIZATION STRATEGY IMPLEMENTATION IN THE REPUBLIC OF MACEDONIA IN 2004-2009}

Kamchev $N^{l}$, Zdravkovska $M^{2}$, Ivanovska $V^{l}$, Kamcheva $G^{l}$ ${ }^{1}$ Faculty of Medical Sciences, University Goce Delcev, Stip, Republic of Macedonia

${ }^{2}$ Medical Faculty, University Ss. Cyril and Methodius, Skopje, Republic of Macedonia

Introduction: The introduction of the vaccines as a protective measure against infectious diseases shows impressive results throughout the history of medicine. Several infectious diseases have been eliminated globally, while many others have been reduced to a negligible amount. The mandatory immunization policy in the Republic of Macedonia has achieved high coverage rates. They are in line with the World Health Organization recommendations and other referent international institutions at levels $\geq 95 \%$, with rare reductions for some vaccines below $95 \%$, and even less occasionally below $90 \%$.

Objectives: To evaluate the implementation of the immunization program in the Republic of Macedonia from 2004 to 2009 , and to present the incidence and prevalence of vaccine-preventable diseases for the same period of time.

Methods and materials: We investigated the number of all registered vaccinated and re-vaccinated people from 2004 to 2009 consistent with the national mandatory immunization policy against certain infectious diseases. The study also included figures on people infected with vaccinepreventable diseases for the same period. The source of relevant information was the Institute of Public Health of the Republic of Macedonia. This was a descriptive epidemiological study.

Results: The coverage rates for primary vaccination against several infectious diseases in 2009 were above $95 \%$. The coverage rate of $96.4 \%$ for the three doses OPV vaccine in 2009 is higher than its average rate of $95.1 \%$ during the period 2004-2008. The coverage rate of $95.9 \%$ for the three doses DTP vaccine in 2009 is $1 \%$ higher than its average rate during the period 2004-2008. The coverage rate of 95.8\% for one dose MMR in 2009 is lower than its average 
rate of $97.8 \%$ during the period 2004-2008. The coverage rates below $95 \%$ for these three vaccines were registered only in 2006.

The coverage rates below $95 \%$ for primary vaccination during the period 2004-2009 were registered for: Hepatitis B vaccine (94.2\%), Haemophilus influenzae type b vaccine (81.5\%) during 2008 and 2009, and for Human Papillomavirus vaccine which was initiated in November 2009. With the exception for MMR, all other re-vaccinations in 2009 are below $95 \%$, but above $90 \%$, which represent lower coverage rates compared with their average rates for the period 2004-2008. From 2004 to
2009, the prevalence of Pertussis was 1.08 per 100,000 inhabitants, Measles 2.45 per 100,000 inhabitants, Mumps 850.6 per 100,000 inhabitants (epidemic in 2008/2009), Rubella 6.68 per 100,000 inhabitants, and Tetanus 0.19 per 100,000 inhabitants.

Conclusions: In Republic of Macedonia, the coverage rates for the majority of vaccines against certain infectious diseases included in the national mandatory immunization policy are well at level of $95 \%$. However, there is a slight tendency of reduction in the coverage rates for primary vaccination, as well as in the re-vaccination rates for certain vaccines in 2009. 